\title{
The modern challenges of haemophilia care in childhood and adolescence
}

\author{
Mary Mathias, Debra Pollard
}

Modern haemophilia care based on good diagnosis and effective prophylaxis has allowed boys born with haemophilia to grow up leading essentially normal lives. Nevertheless, there remain challenges notably those posed by inhibitors and patient expectations. There is now a significant cohort of men in their $30 \mathrm{~s}, 40 \mathrm{~s}$ and $50 \mathrm{~s}$ who have been looked after extremely well but many of whom now have significant ankle arthropathy because they played football, even when advised not to do so at a time when prophylaxis was limited or started late. The imminent era of longer-acting clotting factors and gene therapy will also impact on future patient expectations. Meeting and managing these challenges will be much enhanced by the development and maintenance of good relationships between the patient and the haemophilia team.

Keywords: family, haemophilia, Katharine Dormandy, inhibitors

\section{The consultant perspective ...}

Katharine Dormandy recognised that although only some members of the family were directly affected by haemophilia, indirectly the whole family was affected. This recognition has always been at the centre of the care that families have been given at the Royal Free Hospital.

Working through the 1960s and early 1970s, Katharine Dormandy was able to start delivering effective treatment for boys with severe haemophilia and to move towards home treatment. Through the 1980s and 1990s, her successors were able to establish prophylaxis for boys with severe haemophilia, and at the same time to deal with the consequences of the HIV epidemic. During the 1990s and into the early 21st century, they were able to institute effective prophylaxis using recombinant products and to tailor it in the children they managed.

Today in paediatric haemophilia care we offer a multidisciplinary team approach from diagnosis. We offer alternate-day prophylaxis in haemophilia $A$ and twice-weekly prophylaxis in haemophilia B, to all of our severe patients and some of our moderate patients, with treatment initiated before 2 years of age. Patients have regular physiotherapy and joint scores, not only to manage

Mary Mathias, Consultant Haematologist, Royal Free Hospital and Great Ormond Street Hospital, London

Email: Mary.Mathias@gosh.nhs.uk

Debra Pollard, Lead Nurse, Katharine Dormandy Haemophilia Centre \& Thrombosis Unit, the Royal Free Hospital, London

Email: debra.pollardanhs.net

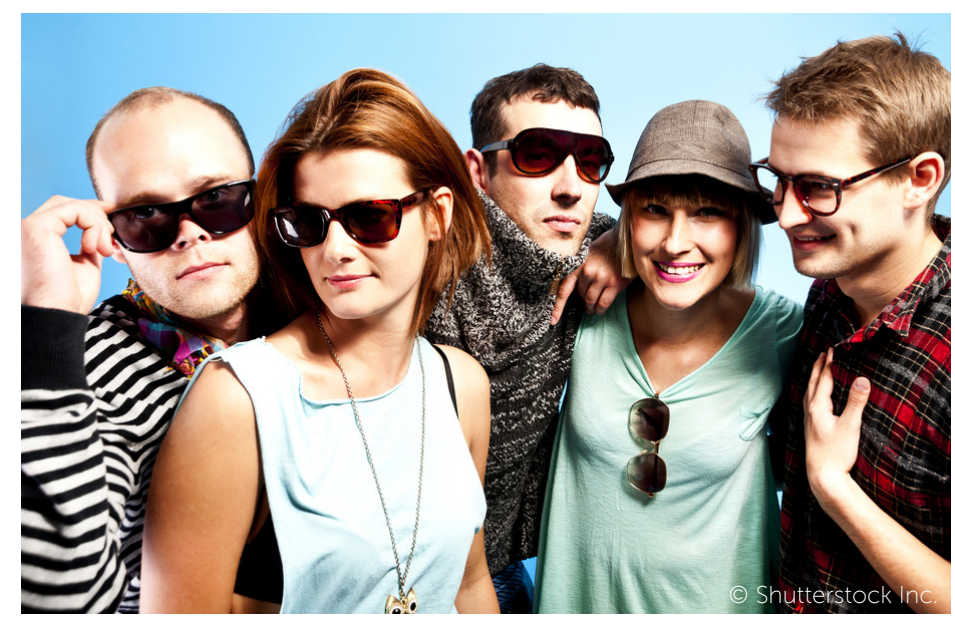

bleeding episodes (most of our patients at Great Ormond Street do not have bleeding episodes); but by doing so we are able to prevent problems from occurring both related to gait disturbance or leg length discrepancy which are, in fact, nothing to do with haemophilia. We are able to encourage them in regular physical activity and we know that they benefit from this in terms of their joint and muscle health. We aim to maintain measurable factor VIII or IX troughs where it is possible to do so, and we encourage most of our boys to be self-treating by the time they are 10 years old and self-managing by their early teens, recognising that there is a small group who will never manage this.

Nevertheless, we are still faced by some challenges. Perhaps the greatest of these is that posed by inhibitors: approximately one in three boys with severe haemophilia A will develop an inhibitor. Analysis of the ethnic breakdown of patients treated at Great Ormond Street born between 2005 and 2015 probably mirrors the ethnic mix of Greater London. However, among that one in three, a much smaller proportion will develop a high titre inhibitor (>10BU at historical peak and requiring more than alternate-day prophylaxis or eradication). The ethnic mix here is very different. As has been well described in the literature, boys who are not Caucasian, and in particular those of Afro-Caribbean descent, have a much greater risk of developing high titre inhibitors. We do not at present know why these boys represent a higher risk of inhibitor formation. But our concern at Great Ormond Street is that they are also less likely to become tolerised. Therefore, we now have a small cohort of seven boys aged 1-14 years who have had between one and five attempts at 


\section{Mary Mathias}

Mary Mathias has been a Consultant in Paediatric Haematology specialising in haemostasis and thrombosis at the Royal Free Hospital since 2006. She also works at Great Ormond Street Hospital as part of the team looking after children with inherited bleeding disorders. Mary's particular interests include management of boys with inhibitors to FVIII and FIX and rare bleeding disorders. Her current research interests include clinical trials of new clotting factor concentrates and registry data collection demonstrating longer term outcomes of regular treatment for children with haemophilia.

immune tolerance, and we have been unable to tolerise them. They are maintained on bypassing agent prophylaxis and of those seven boys, six are non-Caucasian. While we could hope that this might simply be an epidemiological curiosity, given the ethnic mix in London and the country in general, this seems unlikely. Of the four babies and toddlers currently treated at Great Ormond Street, three with inhibitors are non-Caucasian.

This cohort of patients represents a window into the world that Katharine Dormandy must have lived with originally. They are boys who have multiple bleeding episodes and boys who, in some cases, I have had to tell not to go to the schoolyard at playtime. Three of those seven boys are very familiar with a wheelchair. So, unless we are able to offer a paradigm shift in the management of inhibitors, this is a small but very difficult cohort of people whose lives will be significantly limited by this inhibitor.

\section{Patient expectations}

Patient expectations are another issue that we have struggled with over the years in teenage boys born in this country who have had the best haemophilia care available. This is illustrated by three patient scenarios:

\section{- The teenage boy with late diagnosed moderate} haemophilia who regularly takes his prophylaxis but is a very talented at sports and plays at a high level. He will not give it up, despite advice. He has had recurrent haemarthrosis, which has affected his ankle. He will require an arthrodesis or replacement within the next 5-10 years, which will be shortly after he reaches higher education.

- The teenage boy with severe haemophilia diagnosed at birth who's family is unable or unwilling to engage in treatment. He too continues to play sport and now has daily bilateral ankle pain with significant mobility issues and will require a fusion or replacement within the next 5-10 years.

- The teenage boy with severe haemophilia diagnosed at birth who has had extremely good and effective prophylaxis and has not yet had any non-traumatic breakthrough bleeds. He is also a high level footballer, and targets his treatment to his exercise schedule. His ankle joints are in good condition, although scanning suggests some tendonitis, as may be expected.

It is possible to give boys with haemophilia effective prophylaxis so that they mature with essentially pristine joints. But the challenge going forward will be that we will have to achieve this in a world of changing haemophilia care. At the moment, we can advise patients to tailor their prophylaxis, but with the move towards longer-acting products and with boys spending a greater proportion of their time with factor VIII and IX levels under $5 \%$, this will lead to a different picture. We are also moving forward into a world where patients may be converted from severe to moderate haemophilia. At the moment, most patients with moderate haemophilia do not qualify for prophylaxis, unless they have recurrent joint damage and bleeding.

The challenge we will have going forward is how to fit these new treatments into the lives of patients. The reality of haemophilia care when Katharine Dormandy set up this centre was that boys with severe haemophilia spent a significant amount of time in hospital bed in pain. The efforts of all the people who have worked here over the years have meant that physicians today are privileged to experience a very different reality. But with that privilege comes expectations. The expectation of our patients and their families is that boys will be able to engage in the normal level of physical activities of their childhood peers. If they are not in a hospital bed in pain, they will be running around and playing football.

The challenge for physicians is how we advise them. Are we going to say, "We will be able to give you additional factor VIII and IX when you are exercising"? Or will we say to them, "For you, perhaps it would be better to stay on a standard half-life factor VIII, at least in the decades when you are most physically active"? One thing we will not say to them is: "You should stop playing football and take up swimming." This is because we know it does not work. There is now a significant cohort of men in their 30s, 40s and 50s who have been looked after extremely well and who have significant ankle arthropathy because they went out playing football, even when they were told very clearly not to. They played football because they were not in pain. So, this is the reality of what we're going to deal with going forward. I am confident that we will find a way around this as we move into yet another different era in haemophilia care.

\section{The haemophilia nurse perspective...}

Managing haemophilia is a lifetime relationship. It starts with the carrier mother and her relationship with haemophilia. Context and the experience of the family are very important when communicating about haemophilia within a family [1]. It is important to understand:

- What is the mother's relationship with haemophilia?

- What is the wider family's relationship with haemophilia?

- What is the family's relationship with the centre?

- How was the diagnosis made?

- What were the first experiences of hospitals like?

-What were the early experiences of treatment like?

- Did they have difficult venous access at the time of diagnosis and do they have difficult venous access now? All relationships start with good foundations, and for many families there is a lifelong relationship with the 


\section{Debra Pollard}

Debra Pollard is Lead Nurse at the Katharine Dormandy Haemophilia Centre. In September 1991 Debra joined the Haemophilia Centre. While at the Centre, Debra has led the Community Nursing Service and the Home Treatment Training Programme for parents and boys with haemophilia. She has a particular interest in supporting the needs of women with bleeding disorders. Her main academic interests lie in qualitative research, and quality of life in haemophilia.

treatment centre. Therefore, comprehensive care aims to build relationships with boys with haemophilia very early in life. We build the relationships with the carriers and with their extended families and very often their friends too. We have to build relationships with the boys and we have to build relationships with their schools, and this model of community and outreach that Katharine Dormandy published about very early on still continues [2,3]. We talk about their sports and their social activities. So haemophilia nursing is not just about needles and treatments and the technical aspects of care but about making every interaction count. For this reason, at this centre we have organised parties, picnics on Hampstead Heath, parent networks and try to involve brothers and sisters, to make every interaction count.

For most young boys with haemophilia, the first steps towards their independence in their treatment comes when they have their first treatments. We support treatment in the home as well as in the centre, because haemophilia is something that these boys have to live with wherever they are. But the education we provide is around much more than injections, focusing on keeping healthy, how to exercise safely, how to approach some of life's riskier moments. We offer this in workshop settings as well as in one to one sessions because, as we know from Katharine Dormandy's groundwork, it is really important that these boys carry on meeting other people with haemophilia and form supportive peer-based networks. The success of home treatment means that very often these boys do not meet others with haemophilia, unless they have family members with haemophilia.

With or without haemophilia, adolescence is a risky time of life, and there are a number of basic conversations we try to have with our boys with haemophilia:

- Tell your parents who you are with - let them meet your friends

- Wear a Medic-alert or something similar

- Someone in your group should know you have

haemophilia and how to contact your parents

- Alcohol makes you forget your natural inhibitions beware of what you might have to live with when you wake up, and remember that you are more likely to take risks as your judgement is impaired, and more likely to have accidents and injuries.

Unfortunately, having grown up with access to excellent treatment and care, many boys in adolescence start to believe that they are invincible, and they do take unnecessary risks. Risk assessment is something that the haemophilia nurse has to become skilled in, and rather than simply saying "no", we have to find ways to support boys. We have to operate an open-door policy, learn not be shocked by anything we are presented with, and to expect the unexpected, such as the phone call from a boy who say's "I'm halfway up Kilimanjaro, should I have taken an extra dose?"

We have to recognise that boys with haemophilia are primarily boys, and as such they will wish to experience all of the things that other adolescent boys experience. Like their peers, boys with haemophilia will wish to have tattoos. Saying no rarely works in the face of peer pressure: most will go off and do it anyway, so it is far better that we encourage them to do it safely to minimise risk. Body piercing may be very different and requires a case by case approach: for instance, a boy with moderate haemophilia who may not be exposed to regular treatment may be at increased risk of an inhibitor if he is given a dose of treatment in order to have a piercing.

Haemophilia nurses need to be prepared to discuss "difficult issues" such as sex, alcohol and drugs with our boys, as well as the logistical aspects of managing treatment when going to a festival for several days at a time and living in a communal tent. We need to be prepared to work in a multidisciplinary team and to support boys who are struggling with obesity, or those who are particularly worried about body image because they have an underlying condition against which they will sometimes over-compensate, for instance with body building at the risk of excessive muscle bleeds.

Our adolescent haemophilia boys are growing up in a culture that is full of risks. Our role is to encourage them to stay safe while engaging with that world, not to tell them to stay away from it. However difficult it is, in the multidisciplinary team at the Katharine Dormandy Haemophilia Centre, we try to keep the lines of communication with our patients open so that our patients see the centre as a place of safety. Adolescence is normal, but having haemophilia is not normal, and neither is its treatment. Like most adolescents, boys with haemophilia just want to be "normal", whatever that means to them. It is our job to support them.

\section{Disclosures}

The scientific meeting on which this issue of The Journal of Haemophilia Practice is based was sponsored by Baxter, Bayer, CSL Behring, Grifols, Novo Nordisk, Sobi, Pfizer, BPL and Werfen. Editorial support for the article was provided by the publisher.

\section{References}

1. Gregory M, Boddington P, Dimond R, et al. Communicating about haemophilia within the family: the importance of context and of experience. Haemophilia 2007; 13(2): 189-98

2. Britten MI, Spooner RJD, Dormandy KM, Biggs R. The haemophiliac boy in school. Br Med J 1966; 2(5507): 224

3. Le Quesne B, Britten MI, Maragaki C, Dormandy KM. Home treatment for patients with haemophilia. Lancet 1974; 2(7879): 507-9. 